\title{
Isolation and Characterization of Chickpea (Cicer arietinum L.) Nodulating Rhizobia Collected from South Wollo Zone, Ethiopia
}

\author{
Gedefaw Wubie (i) and Mussa Adal $(\mathbb{D}$ \\ Wollo University, College of Natural Science, Department of Biology, Dessie, Amhara Regional State, Ethiopia \\ Correspondence should be addressed to Gedefaw Wubie; wubieg19@gmail.com
}

Received 3 August 2021; Revised 25 September 2021; Accepted 8 October 2021; Published 29 October 2021

Academic Editor: Isabel Marques

Copyright (c) 2021 Gedefaw Wubie and Mussa Adal. This is an open access article distributed under the Creative Commons Attribution License, which permits unrestricted use, distribution, and reproduction in any medium, provided the original work is properly cited.

\begin{abstract}
This study aimed at screening rhizobial isolates of chickpea and evaluating their symbiotic nitrogen fixation efficiency and tolerance to abiotic stresses. A total of $107(100 \%)$ isolates were collected of which 52 (48.6\%) were confirmed as chickpea rhizobia using preliminary tests. Among 52 (48.6\%) isolates, 46 (88.5\%) have induced nodulation on chickpea under greenhouse and were screened under in vitro conditions and $6(11.5 \%)$ of them were discarded due to fail to nodulate. The greenhouse data showed the highest nodule number $\left(68.67\right.$ plant $\left.^{-1}\right)$, nodule dry weight $\left(0.17 \mathrm{~g} \mathrm{plant}^{-1}\right)$, and shoot dry weight $\left(0.81 \mathrm{~g} \mathrm{plant}^{-1}\right)$ were scored by plants inoculated with isolates WUCR 17, WUCR 1, and WUCR 66, respectively. Among authenticated isolates, $73.9 \%, 21.7 \%$, and $4.3 \%$ were highly effective, effective, and lowly effective, respectively. The physiological test showed $15 \%$ of isolates tolerated $13 \%$ salt concentration and $10.9 \%$ of isolates grew at $\mathrm{pH} 4-10$ range. All isolates grew at $20-35^{\circ} \mathrm{C}$ and $13 \%$ grew at maximum temperature $\left(50^{\circ} \mathrm{C}\right)$. The isolates showed better resistance to the tested antibiotics at low concentration $(2.5 \mu \mathrm{g} / \mathrm{ml}) \mathrm{but}$ the majorities were sensitive at high concentration $(10 \mu \mathrm{g} / \mathrm{ml})$. Among the isolates, $13 \%$ tolerated all tested heavy metals but $48 \%$ were sensitive to mercury. Regarding the carbohydrate utilization test, $52.2 \%$ catabolized all the tested 11 carbon sources but $6.5 \%$ of them utilized only $63.6 \%$ carbon sources. Amino acid utilization showed isolates (85\%) utilized D-alanine except WUCR (14, 25, $31,34,39,59$, and 76 ) and $76 \%$ of the isolates utilized arginine and phenylalanine and $74 \%$ utilized leucine as a nitrogen source. Of the isolates, $43.5 \%$ of them utilized both $\mathrm{D}$-alanine and arginine as a nitrogen source. In almost all tests conducted, isolates WUCR 1 and 5 performed top and were recommended as potential candidates for microbial inoculants.
\end{abstract}

\section{Introduction}

Chickpea (Cicer arietinum L.), a member of the family Leguminosae, is an important pulse crop valued as a rich source of proteins [1]. It is a cool-season legume crop that is grown in many countries around the world as a food source [2]. It has an important role in the maintenance of soil fertility, particularly in dry, rain-fed areas, and can resist drought [3]. Chickpea is a staple basic food crop in many tropical and subtropical Afro-Asian countries and is one of the world's major pulse crops which are traditionally cultivated in marginal areas and semiarid regions [4]. Globally, it ranked the third most important pulse crop [5] with an average yield of $913 \mathrm{~kg} / \mathrm{ha}$ and a total production of 10.9 million metric tons [6]. At present, it is produced in over 40 countries of the world of which $89.7 \%$ is in Asia, followed by 4.3\% in Africa, 2.6\% in Oceania, 2.9\% in Americans, and $0.4 \%$ in Europe [7]. About $95 \%$ of chickpea cultivation and consumption is in developing countries [8].

Ethiopia is the first country in chickpea production and production area coverage in Africa [9] where it plays an important role in the local economy and contributes to sustainable agriculture. Currently, chickpea is cultivated in four regions of the country: Amhara, Oromia, Southern Nations Nationalities and People's Region (SNNPR), and Tigray. Amhara and Oromia regions together produce 93\% of total chickpea production in Ethiopia while SNNPR and Tigray produce $3.5 \%$ and $3 \%$, respectively [8]. The top 9 chickpea producing administrative zones (North Gonder, South Gonder, North Shoa, East Gojam, South Wollo, North 
Wollo, West Gojam, and Gonder Zuria) of the Amhara region account for about $80 \%$ of the country chickpea production [10]. However, its yield has remained very low compared to the natural potential of the crop due to several environmental stresses which prevail under Ethiopian edaphoclimatic conditions $[11,12]$.

The importance of selection, commercialization, and application of rhizobia (inoculants) with host pulse crops has been well known for decades [13]. Microbial inoculants are the promising components for integral solutions to agroenvironmental problems because they promote plant growth by enhancing nutrient availability and uptake as well as maintaining the health of the plant [14]. This requires screening compatible hosts, endosymbionts, and appropriate environmental conditions. The inoculation of chickpea seeds with rhizobium is known to increase nodulation, nitrogen uptake, growth, and yield parameters of legume crops and some other biocontrol characteristics [15]. Biofertilization has great importance in decreasing environmental pollution and deterioration of nature [15]. Poor nodulation and the lack of inoculation in field experiments have frequently been reported worldwide, raising doubts about the efficiency of crop inoculation [16-18]. The lack of response to inoculation can be attributed to intrinsic characteristics of both the host plant and the bacteria as well as the great sensitivity of the symbiosis to environmental stresses such as high temperatures, soil dryness, and low soil fertility $[19,20]$.

There are severe problems in soil fertility and crop productions due to excessive use of inorganic fertilizers causing undesirable effects on agriculture, food, biodiversity, and the environment at large [21, 22]. In Ethiopia, low soil fertility and limited external inputs are the major constraints to the low yield of crops [23] particularly low levels of nitrogen and available phosphorus in the country [24]. In agriculture, perhaps $80 \%$ of the biologically fixed nitrogen comes from symbiosis involving leguminous plants and bacteria of the family Rhizobiaceae. Nitrogen, a vitally important plant nutrient, is the main factor that limits chickpea production in Ethiopia. However, these legumes form an effective symbiotic association with a soil-dwelling bacteria known as rhizobia and fix atmospheric nitrogen via biological nitrogen fixation (BNF) process in a plant root structure (nodules). The nitrogen fixed via BNF is mostly used for plant growth and development. This accounts for about $60 \%$ of the global nitrogen budget and is eco-friendly and cost-effective [25]. The symbiotic association that forms BNF is affected by various factors [25] such as the rhizobium strains, host legumes, and biotic and abiotic soil factors.

Researches that focused on the diversity and symbiotic properties of rhizobia and rhizobacteria associated with pulse crops including fava bean, field pea, chickpea, and lentil were carried out $[26,27]$. These studies mainly focused on isolation and in vitro characterization under laboratory conditions and nitrogen fixation under greenhouse conditions [10]. These trials were limited to only a few areas of the country and no literature report in the study area so far [28-32]. The plant growth promoting and controlling role of chickpea rhizobia was forgotten except few works done in other areas outside from the study area and on other crop legumes $[33,34]$. Thus, this study was aimed at isolating and characterization of chickpea (Cicer arietinum L.) nodulating rhizobia collected from South Wollo Zone, Ethiopia.

\section{Materials and Methods}

2.1. Sampling Sites and Sample Collection. The sampling sites were comprised of 40 sampling sites from 4 woredas of South Wollo Zone including Tehuledere, Kutaber, Dessie Zuria, and Kalu (Figure 1), which were selected based on the high chickpea production history and no inoculation of chickpea rhizobia before. The root nodules were collected between October and November 2019 during flowering stage. Pink-colored nodules and $3 \mathrm{~kg}$ of soil were collected from the roots and rhizosphere of a healthy standing plant, respectively. The nodules were collected and placed in sealed vials containing a desiccant (silica gel) covered with $1 \mathrm{~cm}$ of cotton wool and brought to Wollo University Biology Laboratory and kept at $4^{\circ} \mathrm{C}$ till isolation of the rhizobia was conducted [35]. The soil was placed in polythene plastic bags and brought to the laboratory for further work.

\subsection{Isolation of Rhizobia and Purification of the Isolates.} Dehydrated or desiccated root nodules were immersed in sterile distilled water overnight in labeled sets of Petri dishes [36]. The imbibed nodules were surface-sterilized according to [36]. The surface-sterilized nodules were transferred into sterile Petri dishes, crushed, and streaked on Yeast Extract Mannitol Agar containing Congo red (YEMA-CR). The plates were incubated at $28 \pm 2^{\circ} \mathrm{C}$ for 3-5 days and presence and absence of growth were recorded (36).

2.3. Presumptive Test. The growth of the rhizobial isolates was determined by growing them on Peptone Glucose Agar (PGA), Keto-Lactose Test, Gram staining, and YEMA-CR medium $[18,36,37]$. The rhizobia isolates were tested for determining their Gram reaction type using the $\mathrm{KOH}$ method $[33,38]$. The isolates were incubated at $28 \pm 2^{\circ} \mathrm{C}$ for 3-5 days to check the presence and absence of growth. Isolates that showed no growth or very poor growth on glucose peptone agar medium were taken as rhizobia. The media composition includes $(\mathrm{g} / \mathrm{l})$ peptic digest of animal tissue (20), dextrose (10), sodium chloride (5), and agar (15).

2.4. Colony and Growth Characteristics. The morphological characteristics of each isolate were determined according to [39-41]. The isolates were inoculated onto YEMA and incubated at $28 \pm 20^{\circ} \mathrm{C}$ for 3-5 days to determine colony characteristics [40]. Acid-base production was also determined [41]. Their growth was assessed on YEM broth in an Erlenmeyer flask incubated in a Gyratory shaker at 130 revs. $\mathrm{min}^{-1}$ and their doubling time was calculated from the logarithmic phase [42] as

$$
g=\frac{[\log 2(t)]}{[\log X-\log X o]},
$$




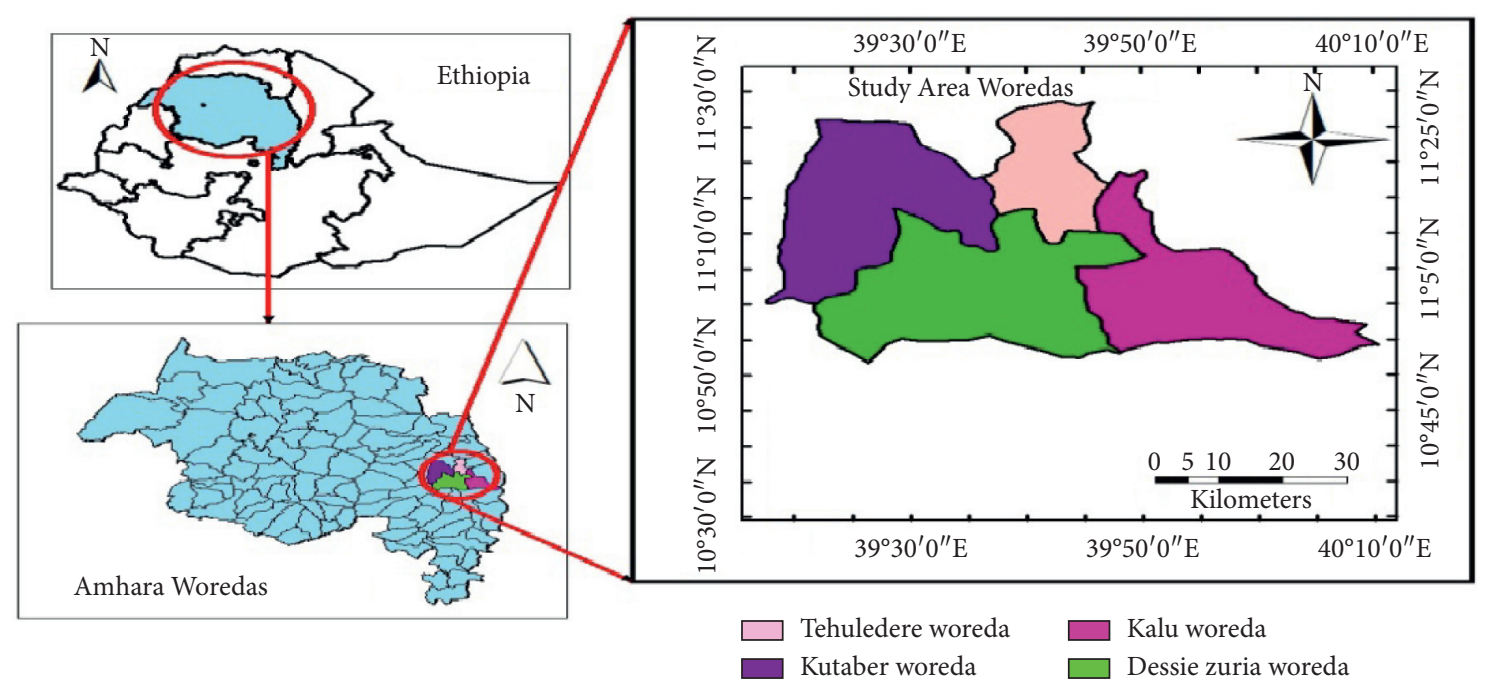

Figure 1: Map of the study area (using Garmin GPS 72H).

where $t$ is the time elapsed, $X o$ is the first OD reading, and $X$ is the second OD reading in the logarithmic phase.

2.5. Greenhouse Authentication of Rhizobia. Pots were washed and surfaced-sterilized and autoclaved as indicated in Somasegaren and Hoben [35]. Chickpea variety called "Desi" was used for the greenhouse experiment. Gray undamaged seeds of uniform size were selected and surface-sterilized and were rinsed in five changes of sterile water and placed to $0.75(\mathrm{w} / \mathrm{v})$ water agar plates. They were incubated at $28 \pm 2^{\circ} \mathrm{C}$ for 3 days. After three days, five germinated seeds were transferred into each surface-sterilized pot for germination which was thin down into three later. After 3 days, each seedling was inoculated with $1 \mathrm{ml}$ of $72 \mathrm{hrs}$ YEMB grown culture. The experiment was statistically laid out with three replications using a completely random design in a greenhouse with a $12 \mathrm{hrs}$ photoperiod and an average of $28^{\circ} \mathrm{C}$ and $15^{\circ} \mathrm{C}$ day and night temperature. The experiment consisted of negative control, nitrogen-fertilization treatment, and bacterial treated treatments. All the plants were fertilized with quarter strength of Broughton and Dilworth $\mathrm{N}$ Free medium per week as described in Somasegaren and Hoben [35]. All the pots were watered every two days.

2.6. Relative Symbiotic Effectiveness of the Rhizobial Isolates. Fifty days after planting, plants were uprooted to record shoot length, nodule number, nodule dry weight, and shoot dry weight. The symbiotic effectiveness (SE) of the isolates was calculated using the formula:

$$
\% \mathrm{SE}=\frac{\text { inoculated plants SDW }}{N-\text { fertilized plants SDW }} \times 100,
$$

$[43,44]$ where SDW = shoot dry weight, $\mathrm{N}=$ nitrogen, and $\mathrm{SE}=$ symbiotic effectiveness. The rate of nitrogen-fixing effectiveness was evaluated as highly effective $\geq 80 \%$, effective $50-79 \%$, lowly effective $35-49 \%$, and ineffective $<35 \%$.

\subsection{Physiological Tolerance Test of Rhizobia}

2.7.1. Salt, $p H$, and Temperature Tolerance of the Isolates. Salt tolerances of the isolates were checked by growing them on YEMA plates containing different concentrations of salt [45]. YEMA was prepared with different concentrations of $\mathrm{NaCl}$ containing $0.5 \%, 1 \%, 2 \%, 3 \%, 4 \%, 5 \%, 6 \%, 7 \%, 8 \%, 9 \%, 10 \%$, $11 \%, 12 \%$, and $13 \%$ of $\mathrm{NaCl}$ as indicated in Bernal and Graham [46]. The $\mathrm{pH}$ tolerance of the isolates was determined by growing them on YEMA adjusted using $0.1 \mathrm{~N} \mathrm{HCl}$ or $\mathrm{NaOH}$ at different $\mathrm{pH}$ values of $4,4.5,5,5.5,6,8,8.5,9,9.5$, and 10 [45]. YEMA medium was used to investigate the growth response of rhizobial isolates to different temperatures by inoculating and incubating the media at $5,10,15,20,35,40,45$, and $50^{\circ} \mathrm{C}$ $[39,47]$. The plates were incubated at $28 \pm 2^{\circ} \mathrm{C}$ for $3-5$ days and the absence and presence of growth were recorded.

2.7.2. Antibiotic and Heavy Metal Resistance Test. The rhizobia isolates were tested for antibiotic sensitivity by Kirby-Bauer disc diffusion method on YEMA agar media $[39,48,49]$ which was amended with the antibiotics ( $\mu \mathrm{g}$ $\mathrm{ml}^{-1}$ ): gentamycin, streptomycin, chloramphenicol, and ampicillin adjusted media supplemented with a concentration of $2.5,5$, and $10[39,49]$. The heavy metal resistance was determined on solid media (YEMA) containing filtersterilized $\mathrm{HM}$ at concentrations $\left(\mu \mathrm{g} \mathrm{ml}^{-1}\right)$ of $\mathrm{Hg}\left(\mathrm{HgCl}_{2}\right)(5)$, $\mathrm{Cr}\left(\mathrm{K}_{2} \mathrm{Cr}_{2} \mathrm{O}_{7}\right)$ (50), $\mathrm{Mn}\left(\mathrm{MnCl}_{2}\right)$ (200), $\mathrm{Ni}\left(\mathrm{NiCl}_{2}\right)(200), \mathrm{Pb}$ $\left(\mathrm{Pb}\left(\mathrm{CH}_{3} \mathrm{COO}\right)_{2}\right)(50)$, and $\mathrm{Zn}\left(\mathrm{ZnCl}_{2}\right)$ (100) according to Abd-Alla et al. [50]. The plates were incubated at $28 \pm 2{ }^{\circ} \mathrm{C}$ for 3-5 days and growth was recorded.

2.7.3. Carbohydrate and Amino Acid Utilization. The carbohydrate utilization test of the rhizobial isolates was evaluated using 11 carbon sources: D-fructose, mannitol, sucrose, lactose, and D-glucose, which are heat-resistant carbohydrates, and galactose, maltose, gelatin, cellulose, and starch, which are heat liable carbon sources, and from sugar alcohol, glycerol was tested [35]. The two different types of amino 
acids which included $\mathrm{D}$-alanine and arginine were used to determine the ability of the isolates to utilize amino acids as indicated in Amarger et al. [51]. The plates were incubated at $28 \pm 2^{\circ} \mathrm{C}$ for $3-5$ days and growth was recorded.

2.8. Data Analysis. The data were analyzed and interpreted using one-way ANOVA. The experimental treatments were compared and contrasted against their control following Duncan's multiple range test (DMRT). The correlation between different parameters was evaluated by using the Pearson correlation coefficient using SPSS version 20 and the dendrogram was constructed using the 65 phenotypic parameters used in the physiological test. The phenotypic parameters were recorded as (1) for the presence of growth and (0) for the absence of growth during the test.

\section{Result}

3.1. Isolation, Presumptive Test, and Cultural Characterization of Rhizobial Isolates. Among a total of 107 (100\%) chickpea nodulating rhizobial isolates, 52 (48.6\%) of them were tentatively confirmed and 55 (51.4\%) failed during preliminary test by conducting YEMA-CR absorption, growth on Peptone-Glucose Agar, and Keto-Lactose media and by their Gram reaction. Authentication work showed that $46(88.5 \%)$ out of the $52(48.6 \%)$ isolates form nodules and were authenticated as chickpea root nodule rhizobia. These isolates were purified and designated as WUCR representing different numbers. The remaining $6(11.5 \%)$ isolates failed to nodulate and were then discarded. Among the 46 isolates, 37 $(80.4 \%)$ of them changed the YEMA-BTB medium into yellow color and the remaining 19.6\% (WUCR 6, 11, 23, 28, $34,55,56,61$, and 82) changed the YEMA-BTB medium into blue which are characteristics of acid and base producing rhizobia. The colony morphology and the cultural characteristics of the isolates showed that $18(39.1 \%)$ of them were large mucoid colonies (LM), 7 (15.2\%) large watery (LW), and $21(45.7 \%)$ large circular with extracellular exopolysaccharide production. Regarding the diameter of the isolates, all of them recorded colony diameter ranging in $2.0-6.0 \mathrm{~mm}$. The largest colony diameter of 6.0 was displayed by isolates WUCR 6,14 , 28 , and 106, whereas the smallest diameter of $2.0 \mathrm{~mm}$ was exhibited by isolates WUCR 2, 31, 33, 61, 82, and 89. The doubling time of the isolates ranged in $1.02-5.14 \mathrm{hrs}$ indicating that all the isolates were fast growers. Generally, the Gram reaction, presumptive test result, the colony morphology, cultural characteristics, and the generation time all showed characteristics of rhizobia and, therefore, the isolates could be tentatively characterized as chickpea rhizobia.

3.2. Symbiotic Effectiveness of Rhizobia Nodulating Chickpea under Greenhouse Conditions. The shoot length, nodule number, nodule dry weight, shoot dry weight, and symbiotic effectiveness data obtained from the greenhouse experiment were presented (Table 1).

The nodulation result revealed that $46(88.5 \%)$ of the isolates induced nodulation upon reinoculation on the host plant. Those isolates showed a significant difference in nodule number, nodule dry weight, and shoot dry weight plant $^{-1}$ at $P<0.05$ level of significance. The highest nodule number ( 68 nodules plant $^{-1}$ ) was scored from isolate WUCR 17 while the smallest number (15 nodules plant $\left.{ }^{-1}\right)$ was recorded from isolate WUCR 37 . Regarding the nodule dry weight, plants inoculated with WUCR 1 accumulated $0.17 \mathrm{~g} /$ $\mathrm{p}$, whereas $0.07 \mathrm{~g} / \mathrm{p}$ nodule dry weight was recorded by plants inoculated with WUCR 14. No nodule developed on control plants (Figure 2$)$. The highest $\left(0.81 \mathrm{~g} \mathrm{plant}^{-1}\right)$ and least $\left(0.277 \mathrm{~g} \mathrm{plant}^{-1}\right)$ shoot dry weight were scored from plants inoculated by isolates WUCR 66 and WUCR 37 and WUCR 101, respectively which was greater than the nitrogen fertilizer treatment $\left(0.583\right.$ plant $\left.^{-1}\right)$ and negative control $\left(0.240 \mathrm{~g} \mathrm{plant}^{-1}\right)$. The symbiotic effectiveness data ranged in 47.6-152.1\% of which 34 (73.9\%), 10 (21.7\%), and 2 (4.3\%) scored highly effective, effective, and low effective, respectively (Table 1).

The highly effective isolates $(34,73.9 \%)$ scored an effective rate at a range of $94.3-152.1 \%$. Among the highly effective isolates, six of them (WUCR 1, WUCR 8, WUCR 15, WUCR 17, WUCR 34, and WUCR 66) scored an effectiveness of 135.6-152.1\%. Ten (21.7\%) isolates showed an effectiveness rate of $58.3-78.1 \%$. The maximum effectiveness recorded by isolate WUCR 1 (152.1\%) was 2.6 times greater than the least effectiveness scored by WUCR 25 (58.3\%). Overall, most of the plants treated with rhizobial isolates produced better plant growth (shoot dry matter) than both positive and negative control plants (Figure 3).

A low, moderate, and strong positive correlation were recorded among the symbiotic parameters at a significant level of 0.01 and 0.05 (Table 2). The correlation recorded between shoot dry weight and symbiotic effectiveness $(r=0.902)$ was strongly positive.

3.3. Pattern of Physiological Tolerance of Isolates. Variation among isolates in their physiological tolerance to $\mathrm{pH}$, temperature, salinity, antibiotics, heavy metals, and nutrient utilization to carbohydrate and amino acid sources was observed.

3.3.1. Tolerance to Salt, $p H$, and Temperature. The isolates showed tolerance and sensitivity to the tested salt concentrations (Figure 4). All the isolates were tolerant to the salt concentration of $0.5 \%$ but showed a steady decrease in growth when they were inoculated into the medium containing 6 to $13 \%$ salt concentration. Consequently, $70 \%$ of the isolates grew at $7 \% \mathrm{NaCl}$, whereas $20 \%$ of isolates were resistant to a salt concentration of $12 \%$. Seven $(15.2 \%)$ of the isolates including WUCR 1, 3, 5, 11, 18, 29, and 31 grew at salt concentration of $13 \%$.

All but five isolates grew at all tested $\mathrm{pH}$ ranges (Figure 5). Isolates WUCR 1, WUCR 5, WUCR 22, WUCR 33, and WUCR 106 showed abundant growth in all the tested $\mathrm{pH}$ values, whereas $41.3 \%$ of the isolates WUCR 2, 3, 8, 9, 15 , $18,23,25,31,34,46,55,56,58,59,62,76,82$, and 89 were sensitive to $\mathrm{pH} 4$ and $\mathrm{pH} 4.5$. Generally, the isolates showed tolerance and sensitivity to $\mathrm{pH}$ range 5.0-10 (Figure 3 ). They grew at both lower and higher $\mathrm{pH}$. 
TABle 1: Performance of selected rhizobia isolates on potted sterilized sand under greenhouse conditions.

\begin{tabular}{|c|c|c|c|c|c|c|c|}
\hline Isolates & Collection sites (Woreda) & $\mathrm{SL} / \mathrm{P}$ & $\mathrm{NN} / \mathrm{P}$ & $\mathrm{NDW} / \mathrm{p}$ & $\mathrm{SDW} / \mathrm{p}$ & SE (\%) & Status \\
\hline WUCR-1 & Tehuledere & $30.7 \pm 2.3^{\mathrm{b}-\mathrm{g}}$ & $56.33 \pm 12.79^{\mathrm{ab}}$ & $0.173 \pm 0.009^{\mathrm{bc}}$ & $0.777 \pm 0.020^{\mathrm{a}--\mathrm{c}}$ & 152.1 & $\mathrm{HE}$ \\
\hline WUCR-2 & Tehuledere & $32.7 \pm 1.2^{\mathrm{c}-\mathrm{h}}$ & $26.00 \pm 2.08^{\mathrm{f}-\mathrm{m}}$ & $0.110 \pm 0.012^{\mathrm{b}}$ & $0.740 \pm 0.029^{\mathrm{a}-\mathrm{d}}$ & 128.9 & $\mathrm{HE}$ \\
\hline WUCR-3 & Tehuledere & $26.2 \pm 1.9^{\mathrm{a}-\mathrm{d}}$ & $38.00 \pm 6.03^{c-j}$ & $0.073 \pm 0.003^{\mathrm{b}}$ & $0.553 \pm 0.075^{\mathrm{c}-1}$ & 98.2 & $\mathrm{HE}$ \\
\hline WUCR-5 & Tehuledere & $30.2 \pm 1.7^{\mathrm{b}-\mathrm{g}}$ & $46.33 \pm 1.45^{\mathrm{b}-\mathrm{e}}$ & $0.100 \pm 0.006^{\mathrm{b}}$ & $0.567 \pm 0.079^{\mathrm{b}-\mathrm{k}}$ & 101.6 & $\mathrm{HE}$ \\
\hline WUCR-6 & Tehuledere & $29.8 \pm 0.6^{\mathrm{b}-\mathrm{g}}$ & $39.33 \pm 2.33^{\mathrm{b}-\mathrm{j}}$ & $0.133 \pm 0.009^{b c}$ & $0.663 \pm 0.073^{\mathrm{a}-\mathrm{e}}$ & 113.6 & $\mathrm{HE}$ \\
\hline WUCR-8 & Tehuledere & $32.2 \pm 2.6^{\mathrm{b}-\mathrm{h}}$ & $51.33 \pm 12.12^{\mathrm{b}-\mathrm{d}}$ & $0.147 \pm 0.015^{\mathrm{bc}}$ & $0.787 \pm 0.099^{\mathrm{a}-\mathrm{c}}$ & 137.8 & $\mathrm{HE}$ \\
\hline WUCR-9 & Tehuledere & $29.0 \pm 0.6^{\mathrm{b}-\mathrm{g}}$ & $51.00 \pm 0.58^{\mathrm{b}-\mathrm{d}}$ & $0.110 \pm 0.015^{\mathrm{b}}$ & $0.563 \pm 0.009^{\mathrm{b}-\mathrm{k}}$ & 98.8 & $\mathrm{HE}$ \\
\hline WUCR-11 & Tehuledere & $30.7 \pm 1.8^{\mathrm{b}-\mathrm{g}}$ & $36.67 \pm 2.85^{\mathrm{d}-1}$ & $0.097 \pm 0.022^{\mathrm{b}}$ & $0.620 \pm 0.105^{\mathrm{a}-\mathrm{h}}$ & 111.3 & $\mathrm{HE}$ \\
\hline WUCR-12 & Tehuledere & $29.5 \pm 1.6^{\mathrm{b}-\mathrm{g}}$ & $19.00 \pm 5.57^{1-\mathrm{m}}$ & $0.087 \pm 0.002^{\mathrm{b}}$ & $0.420 \pm 0.025^{\mathrm{f}-\mathrm{m}}$ & 74.2 & $\mathrm{E}$ \\
\hline WUCR-14 & Tehuledere & $27.7 \pm 1.5^{\mathrm{b}-\mathrm{g}}$ & $23.67 \pm 1.67^{\mathrm{i}-\mathrm{m}}$ & $0.070 \pm 0.006^{\mathrm{b}}$ & $0.360 \pm 0.023^{\mathrm{i}-\mathrm{m}}$ & 76.3 & $\mathrm{E}$ \\
\hline WUCR-15 & Tehuledere & $32.5 \pm 0.8^{\mathrm{c}-\mathrm{h}}$ & $40.67 \pm 3.84^{\mathrm{b}-\mathrm{i}}$ & $0.130 \pm 0.010^{\mathrm{bc}}$ & $0.793 \pm 0.043^{\mathrm{a}-\mathrm{b}}$ & 137.6 & $\mathrm{HE}$ \\
\hline WUCR-16 & Tehuledere & $25.8 \pm 2.1^{\mathrm{a}-\mathrm{c}}$ & $22.33 \pm 2.73^{j-m}$ & $0.070 \pm 0.010^{\mathrm{b}}$ & $0.573 \pm 0.052^{\mathrm{a}-\mathrm{j}}$ & 101 & $\mathrm{HE}$ \\
\hline WUCR-17 & Tehuledere & $32.8 \pm 2.6^{\mathrm{d}-\mathrm{h}}$ & $68.67 \pm 0.88^{\mathrm{a}}$ & $0.123 \pm 0.015^{\mathrm{b}}$ & $0.787 \pm 0.146^{\mathrm{a}-\mathrm{c}}$ & 140.6 & $\mathrm{HE}$ \\
\hline WUCR-18 & Tehuledere & $29.3 \pm 1.7^{\mathrm{b}-\mathrm{g}}$ & $31.33 \pm 4.37^{\mathrm{e}-\mathrm{m}}$ & $0.093 \pm 0.012^{\mathrm{b}}$ & $0.333 \pm 0.028^{b c}$ & 59 & $\mathrm{E}$ \\
\hline WUCR-22 & Kutaber & $33.2 \pm 1.8^{\mathrm{e}-\mathrm{h}}$ & $37.00 \pm 5.51^{\mathrm{d}-\mathrm{k}}$ & $0.117 \pm 0.012^{\mathrm{b}}$ & $0.703 \pm 0.105^{\mathrm{a}-\mathrm{d}}$ & 120.2 & $\mathrm{HE}$ \\
\hline WUCR-23 & Kutaber & $34.2 \pm 2.9^{\mathrm{gh}}$ & $33.67 \pm 6.94^{\mathrm{d}-1}$ & $0.107 \pm 0.007^{\mathrm{b}}$ & $0.680 \pm 0.065^{\mathrm{a}-\mathrm{d}}$ & 116.9 & $\mathrm{HE}$ \\
\hline WUCR-25 & Kutaber & $27.0 \pm 1.0^{\mathrm{b}-\mathrm{f}}$ & $19.67 \pm 3.18^{\mathrm{k}-\mathrm{m}}$ & $0.073 \pm 0.015^{\mathrm{b}}$ & $0.340 \pm 0.040^{\mathrm{k}-\mathrm{m}}$ & 58.3 & $\mathrm{E}$ \\
\hline WUCR-26 & Kutaber & $26.3 \pm 2.0^{\mathrm{a}-\mathrm{e}}$ & $31.67 \pm 7.69^{\mathrm{e}-\mathrm{m}}$ & $0.087 \pm 0.012^{\mathrm{b}}$ & $0.607 \pm 0.092^{\mathrm{a}-\mathrm{h}}$ & 108.7 & $\mathrm{HE}$ \\
\hline WUCR-28 & Kutaber & $31.7 \pm 1.1^{\mathrm{b}-\mathrm{h}}$ & $40.67 \pm 2.96^{\mathrm{b}-\mathrm{i}}$ & $0.097 \pm 0.007^{\mathrm{bc}}$ & $0.727 \pm 0.048^{\mathrm{a}-\mathrm{d}}$ & 126.9 & $\mathrm{HE}$ \\
\hline WUCR-29 & Kutaber & $30.7 \pm 2.0^{\mathrm{b}-\mathrm{g}}$ & $36.67 \pm 2.73^{\mathrm{d}-1}$ & $0.113 \pm 0.019^{\mathrm{b}}$ & $0.533 \pm 0.033^{\mathrm{d}-1}$ & 94.3 & $\mathrm{HE}$ \\
\hline WUCR-30 & Kutaber & $25.8 \pm 1.3^{\mathrm{a}-\mathrm{c}}$ & $37.00 \pm 1.16^{\mathrm{d}-\mathrm{k}}$ & $0.087 \pm 0.003^{\mathrm{b}}$ & $0.347 \pm 0.033^{\mathrm{j}-\mathrm{m}}$ & 59.8 & $\mathrm{E}$ \\
\hline WUCR-31 & Kutaber & $28.7 \pm 1.5^{\mathrm{b}-\mathrm{g}}$ & $24.33 \pm 4.41^{\mathrm{g}-\mathrm{m}}$ & $0.089 \pm 0.005^{b c}$ & $0.360 \pm 0.038^{\mathrm{i}-\mathrm{m}}$ & 63.4 & $\mathrm{E}$ \\
\hline WUCR-33 & Kutaber & $30.3 \pm 0.3^{\mathrm{b}-\mathrm{g}}$ & $36.33 \pm 6.69^{\mathrm{d}-1}$ & $0.100 \pm 0.015^{\mathrm{b}}$ & $0.770 \pm 0.084^{\mathrm{a}-\mathrm{d}}$ & 133.1 & $\mathrm{HE}$ \\
\hline WUCR-34 & Kutaber & $29.3 \pm 0.7^{\mathrm{b}-\mathrm{g}}$ & $41.67 \pm 4.91^{\mathrm{b}-\mathrm{h}}$ & $0.107 \pm 0.020^{\mathrm{b}}$ & $0.777 \pm 0.044^{\mathrm{a}-\mathrm{c}}$ & 135.6 & $\mathrm{HE}$ \\
\hline WUCR-35 & Kutaber & $31.7 \pm 1.2^{\mathrm{b}-\mathrm{h}}$ & $40.33 \pm 3.48^{\mathrm{b}-\mathrm{i}}$ & $0.133 \pm 0.022^{b c}$ & $0.710 \pm 0.053^{\mathrm{a}-\mathrm{d}}$ & 122.9 & $\mathrm{HE}$ \\
\hline WUCR-37 & Kutaber & $25.3 \pm 0.7^{\mathrm{ab}}$ & $14.67 \pm 4.84^{\mathrm{mn}}$ & $0.087 \pm 0.003^{\mathrm{b}}$ & $0.277 \pm 0.024^{c}$ & 47.6 & LE \\
\hline WUCR-39 & Kutaber & $28.3 \pm 3.3^{\mathrm{b}-\mathrm{g}}$ & $43.67 \pm 7.31^{\mathrm{b}-\mathrm{f}}$ & $0.120 \pm 0.029^{\mathrm{b}}$ & $0.590 \pm 0.150^{\mathrm{a}-\mathrm{i}}$ & 107.4 & $\mathrm{HE}$ \\
\hline WUCR-46 & Kutaber & $28.3 \pm 1.2^{\mathrm{b}-\mathrm{g}}$ & $38.33 \pm 2.60^{c-j}$ & $0.103 \pm 0.003^{\mathrm{b}}$ & $0.407 \pm 0.015^{\mathrm{g}-\mathrm{m}}$ & 70.9 & $\mathrm{E}$ \\
\hline WUCR-49 & Kutaber & $32.5 \pm 1.3^{\mathrm{c}-\mathrm{h}}$ & $37.67 \pm 3.28^{\mathrm{c}-\mathrm{j}}$ & $0.090 \pm 0.012^{\mathrm{b}}$ & $0.727 \pm 0.073^{\mathrm{a}-\mathrm{d}}$ & 128.1 & $\mathrm{HE}$ \\
\hline WUCR-51 & Kutaber & $30.7 \pm 0.7^{\mathrm{b}-\mathrm{g}}$ & $26.00 \pm 3.61^{f-m}$ & $0.090 \pm 0.000^{\mathrm{b}}$ & $0.427 \pm 0.015^{\mathrm{f}-\mathrm{m}}$ & 74.3 & $\mathrm{E}$ \\
\hline WUCR-55 & Kutaber & $29.0 \pm 0.6^{\mathrm{b}-\mathrm{g}}$ & $40.67 \pm 2.60^{\mathrm{b}-\mathrm{i}}$ & $0.110 \pm 0.006^{\mathrm{b}}$ & $0.670 \pm 0.068^{\mathrm{a}-\mathrm{e}}$ & 117.8 & $\mathrm{HE}$ \\
\hline WUCR-56 & Kutaber & $37.5 \pm 9.0^{\mathrm{h}}$ & $28.33 \pm 8.99^{\mathrm{f}-\mathrm{m}}$ & $0.087 \pm 0.033^{\mathrm{b}}$ & $0.400 \pm 0.038^{\mathrm{h}-\mathrm{m}}$ & 69.4 & $\mathrm{E}$ \\
\hline WUCR-57 & Kutaber & $33.0 \pm 0.0^{\mathrm{d}-\mathrm{h}}$ & $28.00 \pm 2.00^{\mathrm{f}-\mathrm{m}}$ & $0.610 \pm 0.255^{\mathrm{a}}$ & $0.713 \pm 0.043^{\mathrm{a}-\mathrm{d}}$ & 123.6 & $\mathrm{HE}$ \\
\hline WUCR-58 & Kutaber & $29.0 \pm 0.6^{\mathrm{b}-\mathrm{g}}$ & $38.67 \pm 2.85^{\mathcal{c}-j}$ & $0.100 \pm 0.006^{\mathrm{b}}$ & $0.633 \pm 0.023^{\mathrm{a}-\mathrm{g}}$ & 111 & $\mathrm{HE}$ \\
\hline WUCR-59 & Kutaber & $33.5 \pm 0.5^{\mathrm{f}-\mathrm{h}}$ & $34.33 \pm 4.41^{\mathrm{d}-1}$ & $0.130 \pm 0.006^{\mathrm{bc}}$ & $0.713 \pm 0.049^{\mathrm{a}-\mathrm{d}}$ & 125.5 & $\mathrm{HE}$ \\
\hline WUCR-61 & Kutaber & $27.8 \pm 0.9^{\mathrm{b}-\mathrm{g}}$ & $24.00 \pm 1.00^{\mathrm{h}-\mathrm{m}}$ & $0.077 \pm 0.009^{\mathrm{b}}$ & $0.443 \pm 0.055^{\mathrm{e}-\mathrm{m}}$ & 78.1 & E \\
\hline WUCR-62 & Kutaber & $30.0 \pm 0.0^{\mathrm{b}-\mathrm{g}}$ & $42.00 \pm 5.00^{\mathrm{b}-\mathrm{g}}$ & $0.100 \pm 0.025^{\mathrm{b}}$ & $0.593 \pm 0.032^{\mathrm{a}-\mathrm{h}}$ & 103.2 & $\mathrm{HE}$ \\
\hline WUCR-65 & Kutaber & $29.3 \pm 0.7^{\mathrm{b}-\mathrm{g}}$ & $30.00 \pm 8.08^{\mathrm{e}-\mathrm{m}}$ & $0.120 \pm 0.006^{\mathrm{b}}$ & $0.557 \pm 0.062^{\mathrm{b}-1}$ & 99.3 & $\mathrm{HE}$ \\
\hline WUCR-66 & Kutaber & $32.8 \pm 0.6^{\mathrm{d}-\mathrm{h}}$ & $54.67 \pm 3.71^{\mathrm{a}-\mathrm{c}}$ & $0.127 \pm 0.013^{\mathrm{b}}$ & $0.810 \pm 0.070^{\mathrm{a}}$ & 141.7 & $\mathrm{HE}$ \\
\hline WUCR-74 & Kutaber & $31.7 \pm 1.2^{\mathrm{b}-\mathrm{h}}$ & $32.00 \pm 9.02^{\mathrm{e}-\mathrm{m}}$ & $0.107 \pm 0.015^{\mathrm{b}}$ & $0.700 \pm 0.103^{\mathrm{a}-\mathrm{d}}$ & 121.6 & $\mathrm{HE}$ \\
\hline WUCR-76 & Kutaber & $32.0 \pm 1.2^{\mathrm{b}-\mathrm{h}}$ & $34.33 \pm 2.03^{\mathrm{d}-1}$ & $0.133 \pm 0.022^{b c}$ & $0.560 \pm 0.071^{\mathrm{b}-1}$ & 99.8 & $\mathrm{HE}$ \\
\hline WUCR-82 & Kutaber & $29.0 \pm 1.0^{\mathrm{b}-\mathrm{g}}$ & $34.00 \pm 4.36^{\mathrm{d}-1}$ & $0.103 \pm 0.009^{\mathrm{b}}$ & $0.663 \pm 0.118^{\mathrm{a}-\mathrm{e}}$ & 112.2 & $\mathrm{HE}$ \\
\hline WUCR-89 & Kutaber & $31.7 \pm 2.2^{\mathrm{b}-\mathrm{h}}$ & $35.00 \pm 6.66^{\mathrm{d}-1}$ & $0.113 \pm 0.024^{\mathrm{b}}$ & $0.580 \pm 0.035^{\mathrm{a}-\mathrm{i}}$ & 101.3 & $\mathrm{HE}$ \\
\hline WUCR-101 & Kutaber & $27.7 \pm 1.3^{\mathrm{b}-\mathrm{g}}$ & $22.33 \pm 2.40^{\mathrm{j}-\mathrm{m}}$ & $0.291 \pm 0.210^{\mathrm{b}}$ & $0.277 \pm 0.020^{c}$ & 48.7 & LE \\
\hline WUCR-105 & Kutaber & $31.3 \pm 1.2^{\mathrm{b}-\mathrm{h}}$ & $31.67 \pm 5.70^{\mathrm{e}-\mathrm{m}}$ & $0.113 \pm 0.015^{\mathrm{b}}$ & $0.680 \pm 0.132^{\mathrm{a}-\mathrm{d}}$ & 115.9 & $\mathrm{HE}$ \\
\hline WUCR-106 & Kutaber & $28.5 \pm 1.0^{\mathrm{b}-\mathrm{g}}$ & $36.67 \pm 1.76^{\mathrm{d}-1}$ & $0.103 \pm 0.009^{\mathrm{b}}$ & $0.643 \pm 0.033^{\mathrm{a}-\mathrm{f}}$ & 112.7 & $\mathrm{HE}$ \\
\hline - Cont. & - & $20.5 \pm 0.8^{\mathrm{a}}$ & $0.00 \pm 0.00^{\mathrm{n}}$ & $0.000 \pm 0.000^{c}$ & $0.240 \pm 0.040^{\mathrm{c}}$ & 40.9 & - \\
\hline + Cont. & - & $27.3 \pm 0.3^{\mathrm{b}-\mathrm{g}}$ & $0.00 \pm 0.00^{\mathrm{n}}$ & $0.000 \pm 0.000^{c}$ & $0.583 \pm 0.058^{\mathrm{a}-\mathrm{i}}$ & 100 & - \\
\hline Total & - & $29.9 \pm 0.3$ & $34.10 \pm 1.19$ & $0.115 \pm 0.009$ & $0.583 \pm 0.015$ & 102.5 & - \\
\hline
\end{tabular}

$\mathrm{SL} / \mathrm{p}=$ shoot length per plant, $\mathrm{NN} / \mathrm{p}=$ module number per plant, $\mathrm{NDW} / \mathrm{p}=$ nodule dry weight per plant, $\mathrm{SDW} / \mathrm{p}=$ shoot dry weight per plant, $\mathrm{RE}=$ relative effectiveness, $\mathrm{E}=$ effective, $\mathrm{LE}=$ lowly effective, $\mathrm{HE}=$ highly effective, $\mathrm{HE}>80 \%, E 50-80 \%$, LE $35-50 \%$, IE $<35 \%$, the same letters are not significantly different at $P<0.05$ level, and all the data are the means of triplicates. The unit for measuring SL/p is centimeter and the other NDW/p and SDW/p are in gram.

The isolates showed growth at different temperature ranges (Figure 6). All isolates grew at a temperature range of $20-35^{\circ} \mathrm{C}$, whereas $41.3 \%$ showed a wide range of temperature tolerance. A progressive decrease in growth of isolates at temperature ranges of $35^{\circ} \mathrm{C}$ to $50^{\circ} \mathrm{C}$ was observed. Isolates WUCR 5, 16, 22, 25, 26, 30, 46, 49, and 65 grew at all tested incubation temperatures $\left(5-50^{\circ} \mathrm{C}\right)$.
3.3.2. Resistance and Sensitivity to Antibiotics and Heavy Metals. The isolates showed resistance and sensitivity to different antibiotics at different concentrations (Figure 7). The isolates were sensitive (58\%) and resistant (98\%) to chloramphenicol at $10 \mu \mathrm{g} / \mathrm{ml}$ and $2.5 \mu \mathrm{g} / \mathrm{ml}$ concentrations, respectively. Thirteen $(28 \%)$ of the isolates were highly resistant to different antibiotics at different concentrations. 


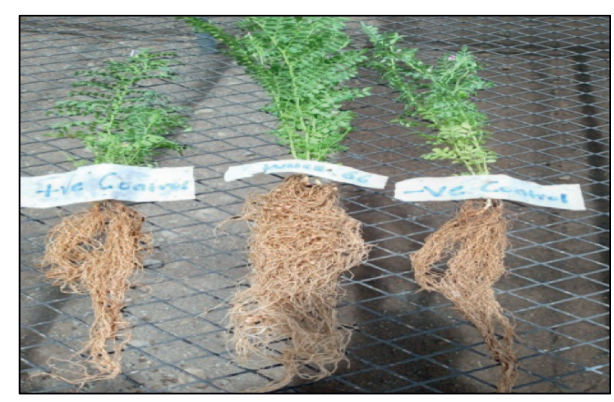

FIgUre 2: Effect of rhizobial inoculation on nodulation.

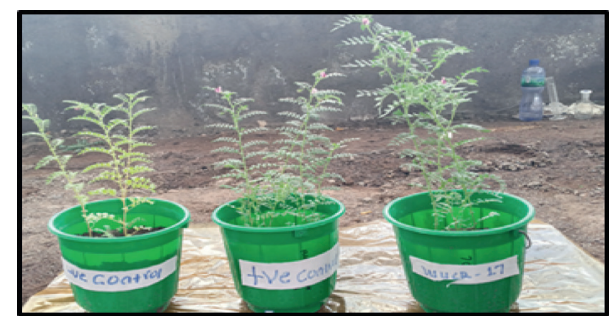

Figure 3: Effect of rhizobia on shoot growth.

TABle 2: Pearson correlation coefficient comparisons for nodule number, nodule dry weight, shoot dry weight, shoots length, and symbiotic effectiveness.

\begin{tabular}{lccccc}
\hline \multicolumn{5}{c}{ Correlations } \\
& $\mathrm{SE}$ & $\mathrm{SDW} / \mathrm{p}$ & $\mathrm{NDW} / \mathrm{p}$ & $\mathrm{NN} / \mathrm{p}$ & $\mathrm{SL} / \mathrm{P}$ \\
\hline $\mathrm{SE}$ & 1 & $0.902^{* *}$ & 0.155 & $0.531^{* *}$ & $0.485^{* *}$ \\
$\mathrm{SDW} / \mathrm{p}$ & $0.902^{* *}$ & 1 & $0.189^{*}$ & $0.548^{* *}$ & $0.474^{* *}$ \\
$\mathrm{NDW} / \mathrm{p}$ & 0.155 & $0.189^{*}$ & 1 & 0.148 & $0.215^{* *}$ \\
$\mathrm{NN} / \mathrm{p}$ & $0.531^{* *}$ & $0.548^{* *}$ & 0.148 & 1 & $0.385^{* *}$ \\
$\mathrm{SL} / \mathrm{P}$ & $0.485^{* *}$ & $0.474^{* *}$ & $0.215^{* *}$ & $0.385^{* *}$ & 1 \\
\hline
\end{tabular}

${ }^{* *}$ Correlation is significant at the 0.01 level (2-tailed). ${ }^{*}$ Correlation is significant at the 0.05 level (2-tailed). $\mathrm{NN} / \mathrm{p}=$ nodule number per plant, $\mathrm{NDW} / \mathrm{p}=$ nodule dry weight, $\mathrm{SDW} / \mathrm{P}=$ shoot dry weight per plant, SL/ $\mathrm{p}=$ shoot length per plant, $\mathrm{SE}=$ symbiotic effectiveness.

The isolates showed variation in the tolerance and sensitivity to different antibiotics at different levels of concentrations.

The isolates were resistant to chromium (74\%), nickel (67\%), and lead (65\%), fairly resistant to manganese (59\%) and zinc (54\%), but sensitive to mercury (48\%). However, $13 \%$ of the isolates (WUCR 2, 5, 9, 33, 35, and 105) tolerated all the tested heavy metals (Figure 8).

3.3.3. Carbohydrate and Amino Acid Utilization. All the isolates catabolized a large variety of carbon sources (Figure 9). The isolates catabolized D-glucose (100\%), D-fructose $(83 \%)$, maltose $(100 \%)$, lactose $(91 \%)$, sucrose $(91 \%)$, cellulose $(78 \%)$, starch $(78 \%)$, galactose $(96 \%)$, gelatin $(91 \%)$, mannitol (100\%), and glycerol (98\%). Of the isolates, $52.2 \%$ grew on the basal medium containing all the 11 tested carbon sources while isolates WUCR 2, 5, 25, 28, 30, 33, and 46 showed abundant growth on all tested carbon sources which is a property of fast-growing rhizobia.

The majority of isolates utilized the four tested amino acid substrates as sources of nitrogen (Figure 10). Most of the isolates (85\%) utilized D-alanine except WUCR 14, 25, $31,34,39,59$, and 76 , and $76 \%$ of the isolates utilized arginine and phenylalanine and $74 \%$ utilized leucine as a nitrogen source. Of the isolates, $43.5 \%$ utilized both $\mathrm{D}$-alanine and arginine.

3.3.4. Numerical Analysis. The phenotypic classes of all isolates are divided into four main clusters with several subclusters based on the 65 phenotypic features at $25 \%$ dissimilarity level (Figure 11). The first cluster (Cluster-I) contained a total of 26 isolates grouped under 2 subclusters IA (19) and further divided into two, IA1 (16) isolates (WUCR 101, 51, 31, 18, 15, 56, 34, 89, 62, 37, 35, 49, 106, 22, 5 , and 1) and IA2 (3) isolates (WUCR 3, 11, and 29), and the other subcluster IB (7) isolates are grouped into two, 3 (WUCR 55, WUCR 74, and WUCR 82) of them with one group at $5 \%$ dissimilarities and the second containing 4 (33, 39,105 , and 57) with the same group. Cluster II only contained one isolate (WUCR 65). Cluster III contained 16 isolates that were divided into two subclusters (C-IIIA and C-IIIB) with WUCR-15 and WUCR - 1 isolates, respectively. The last cluster (C-IV) contained 3 isolates that are divided into two groups (IVA and IVB) with WUCR 2 and WUCR 1 isolates, respectively. Clustering decreased below 5\% level of dissimilarity indicating their phenotypic diversity.

\section{Discussion}

In this study, among 107 root nodule bacteria collected from chickpea, 52 (48.6\%) of them did not absorb Congo red, did not grow on PGA media, and were Gram-negative, which were characteristic features of rhizobia. This result was similar to earlier works reported by Daniel Muleta [30] and Jida and Assefa [27] implying the isolates could be chickpea rhizobia. The isolates changed YEMA-BTB into yellow $(80.4 \%)$ and blue $(19.6 \%)$ which was similarly reported by Jordan [41] and Lupwayi and Haque [39] indicating that they were fast (acid producers) and slow (base producers) growing rhizobia [52]. Regarding growth rate, 10 (22\%), 29 (63\%), and 7 (15\%) showed doubling time of $<2 \mathrm{hrs}, 2-4 \mathrm{hrs,}$ and $4-5.14 \mathrm{hrs}$, respectively, which was in line with the findings of Jida and Assefa [27] who reported doubling time of fewer than $6.0 \mathrm{hrs}$. The isolates are large mucoid (39.1\%), large watery $(15.2 \%)$, and large circular (15.2 with extracellular exopolysaccharide production as described by earlier authors [27, 32, 34, 53-55]. Moreover, the isolates exhibited a colony diameter ranging in $2-6 \mathrm{~mm}$ which was similarly reported $[53,54]$. All the presumptive tests, colony, and cultural characteristics were characteristics of rhizobia indicating that the isolates can be categorized as chickpea rhizobia and previous studies in other locations have established that most chickpea rhizobia belong to $M$. ciceri or M. mediterraneum $[52,56]$.

Among the isolates subjected to greenhouse authentication, $46(89 \%)$ were authenticated as root nodule rhizobia, whereas $6(11 \%)$ failed to nodulate which showed better nodulation compared to Daniel Muleta [30] who reported $47 \%$ nodulation failure that can be related to the inherent links 


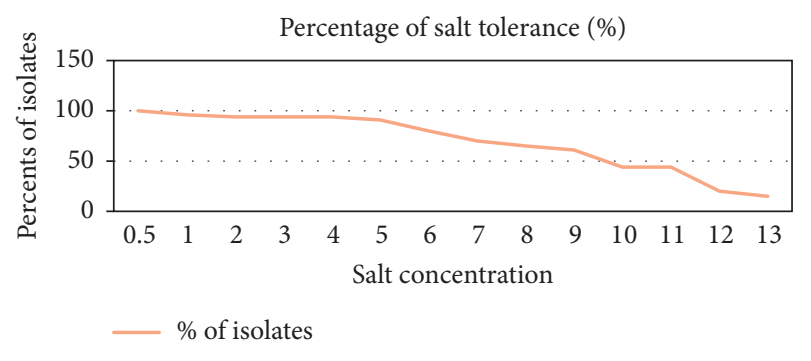

Figure 4: Salt tolerance of chickpea rhizobia.

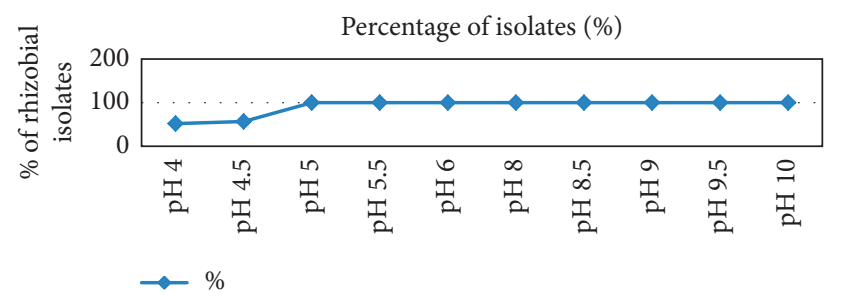

Figure 5: Tolerance of rhizobia isolates to different $\mathrm{pH}$ levels.

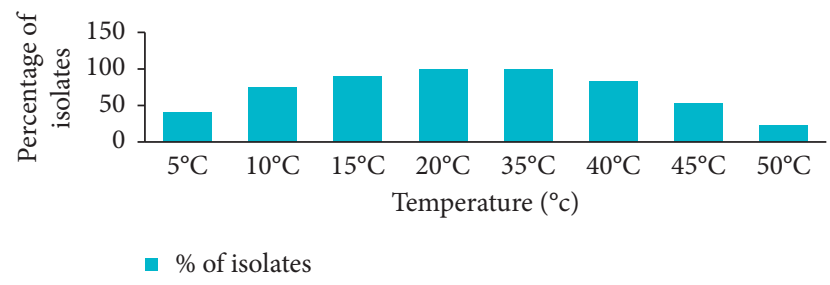

FIgURE 6: Temperature tolerance of chickpea rhizobia.

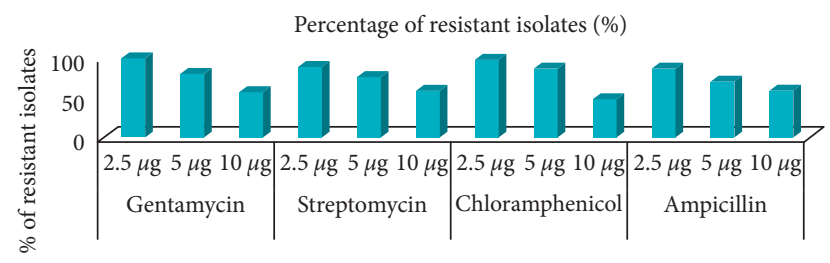

FIgURE 7: Antibiotics tolerance of chickpea rhizobial isolates.

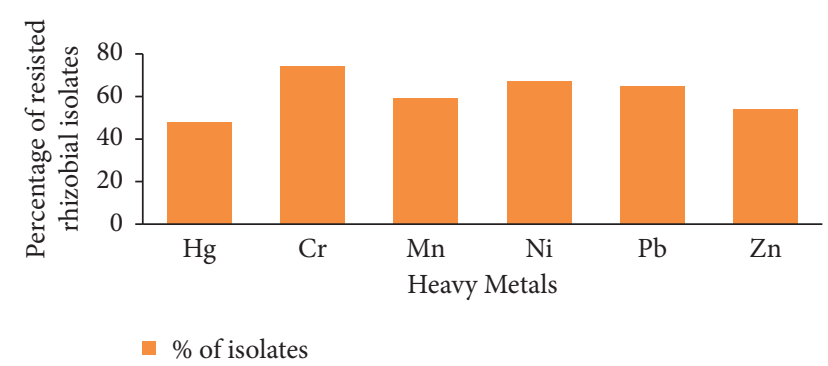

FIGURE 8: Heavy metal resistance of chickpea rhizobia isolates.

between the rhizobia and the hosts' capacity to fix nitrogen [57]. The highest nodule number ( 68 plant $\left.^{-1}\right)$ was scored from isolate WUCR 17 and the least number $\left(15\right.$ plant $\left.^{-1}\right)$ from isolate WUCR 37 which is nearly similar to the findings of Tadele Ereso [32] and Mulisa Jida and Fassil Assefa [27] who reported a maximum and minimum of 66,79 , and 7.5 , and 41

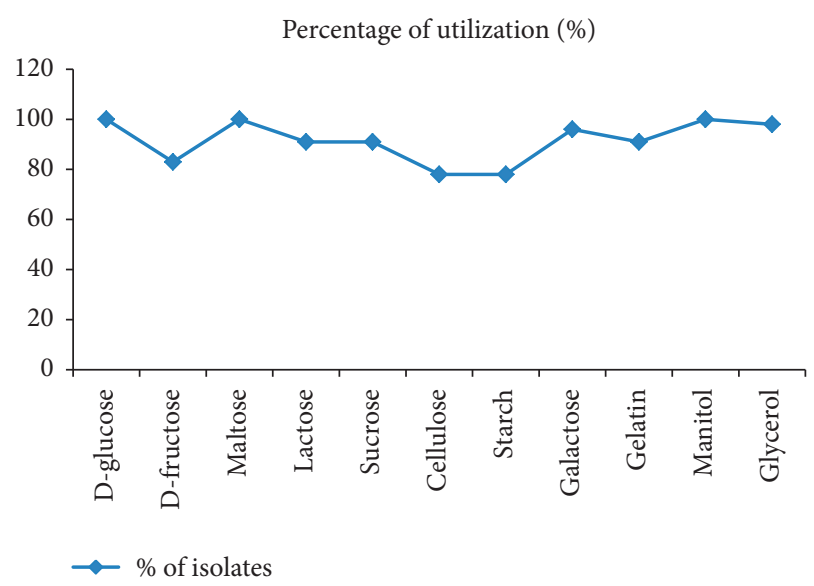

FIGURE 9: Utilizations of different carbon sources of chickpea rhizobia isolates.

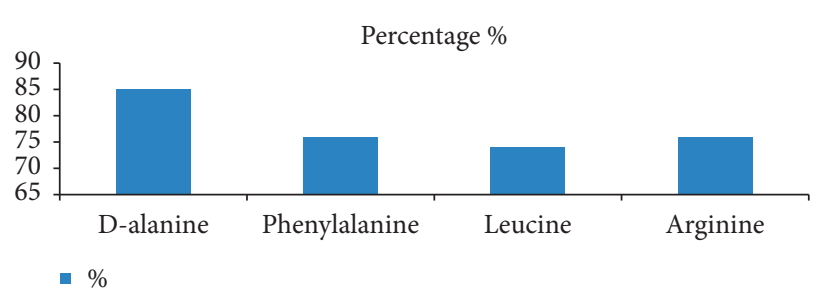

FIgUre 10: Amino acid utilization of chickpea rhizobial isolates.

nodule numbers per plant, respectively. Maatallah et al. [1] similarly reported nodule numbers ranging from 11 to 62 indicating agroecological zone is a major factor for rhizobia nodulation and nitrogen fixation. Plants inoculated by WUCR 1 and WUCR 14 produced maximum and minimum nodule dry weight of $0.17 \mathrm{~g} / \mathrm{p}$ and $0.070 \mathrm{~g} / \mathrm{p}$ which was less as compared to the result of Jida and Assefa [27] who reported $1.16 \mathrm{~g} /$ $\mathrm{p}$ and $0.56 \mathrm{~g} / \mathrm{p}$ as maximum and minimum nodule dry weight, respectively. The highest shoot dry weight $\left(0.81 \mathrm{~g} \mathrm{plant}^{-1}\right)$ was scored from plants inoculated by isolate WUCR 66 which was greater than the nitrogen-fertilization treatment $(0.583$ plant $\left.^{-1}\right)$ whereas the least mean shoot dry weight $(0.277 \mathrm{~g}$ plant $^{-1}$ ) was obtained from plant inoculated with WUCR 37 and WUCR 101 which was greater than the negative control $\left(0.240 \mathrm{~g} \mathrm{plant}^{-1}\right)$. The result of shoot dry weight of this study was still less than the result reported by Jida and Assefa [27] that accumulated shoot dry weight of $0.60 \mathrm{~g} / \mathrm{p}$ and $1.36 \mathrm{~g} / \mathrm{p}$ indicating variation in the nitrogen-fixing capacity which could be due to difference in rhizobial strain type and other soil factors [58]. The nodule dry weight directly correlated positively with the shoot dry weight which is a good indicator in determining factors of nitrogen fixation $[55,59]$.

The isolates showed symbiotic effectiveness ranging in $47.6-152.1 \%$ of which $73.9 \%, 21.7 \%$, and $4.3 \%$ were highly effective, effective, and low effective, respectively. Jida and Assefa [27] reported $11 \%$ highly effective chickpea rhizobia. Similarly, Jida and Assefa [26] and Jida and Assefa [60] reported $33 \%$ and $24 \%$ highly effective rhizobia isolated from lentil and faba bean, respectively. The strong positive correlation between shoot dry weight and symbiotic 


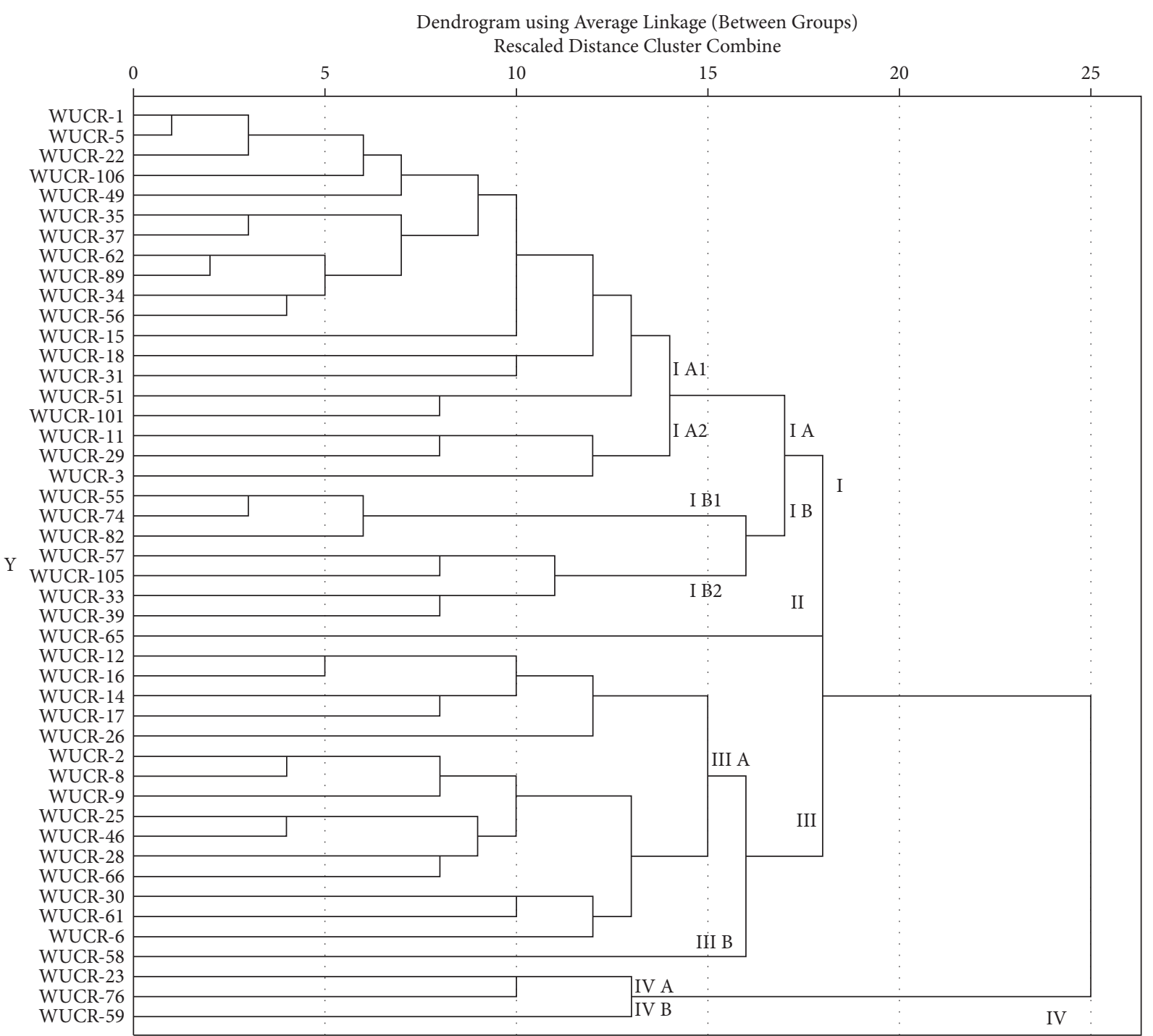

FIGURE 11: A Dendrogram highlighting the phenotypic diversity of chickpea rhizobial isolates.

effectiveness $(r=0.902)$ showed more linear relationships than other parameters indicating that shoot dry weight is a good indicator of symbiotic effectiveness which is an important parameter for determining the symbiotic effectiveness of legume nodulating rhizobia [59].

In this study, all the isolates tolerated the tested $\mathrm{pH}$ at a range of $5-10$ which was slightly similar to $[54,61]$ which reported a $\mathrm{pH}$ range of 4.5 to 8.5 and [62] $\mathrm{pH}$ 6-10 implying that the isolates of this study grew at both lower and higher $\mathrm{pH}[1,53,63]$ which is an indication that chickpea rhizobia exhibit moderately acidic and alkaline characteristics. The isolates tolerated higher concentrations of salt $(0.5-13 \%)$ which is not reported by earlier works $[1,64]$ indicating that the isolates can be applied in areas of high salinity. Although there is variation, the isolates showed growth at both lower and higher temperature ranges $\left(5-50^{\circ} \mathrm{C}\right)$ which is closely similar to the findings of $[1,65,66]$ indicating that the isolates can be applied in areas of low and high temperature [25].

The variation in the tolerance and sensitivity of the isolates to different concentrations could help for better coexistence with antibiotic-producing soil microorganisms in the soil. The occurrence of resistant isolates to the tested heavy metals could be important to improve crop yield when inoculated in soils contaminated with toxic heavy metals [63]. All of the isolates catabolized a large variety of carbon sources that agreed with [27] which described that rhizobia catabolize a wide range of substrates and could have an ecological advantage which is a property of fast-growing rhizobia [41]. The isolates utilized four different amino acid substrates as sources of nitrogen that agreed with $[64,67]$ which reported most of the nitrogen sources were utilized by rhizobia nodulating chickpea. This utilization of carbon and nitrogen sources could be used as diagnostic features for the identification of the rhizobia and is important to improve their survival advantage [68].

The numerical analysis conducted based on 65 phenotypic traits grouped the isolates into four main clusters with several subclusters at 25\% dissimilarity level (Figure 11). Clusters I, II, III, and IV comprised 26, 1, 16, and 3 isolates, respectively. Clustering decreased below 5\% level of 
dissimilarity indicating their phenotypic diversity. All taken together, the clusters indicated high diversity among chickpea rhizobial isolates. The dendrogram constructed grouped the study isolates into different clusters indicating their ecological diversity and relatedness [55]. In this study, chickpea rhizobial isolates endowed with multiple plant growth promoting and biocontrolling properties which could be applied as field conditions were screened.

\section{Data Availability}

The data used to support the findings of this study are available from the corresponding author upon request.

\section{Conflicts of Interest}

The authors declare that there exist no conflicts of interest.

\section{Acknowledgments}

This work was supported by funds from Wollo University, Dessie, Ethiopia.

\section{References}

[1] J. Maatallah, E. B. Berraho, S. Munoz, J. Sanjuan, and C. Lluch, "Phenotypic and molecular characterization of chickpea rhizobia isolated from different areas of Morocco," Journal of Applied Microbiology, vol. 93, no. 4, pp. 531-540, 2002.

[2] J. D. Berger and N. C. Turner, "The ecology of chickpea: evolution, distribution, stresses and adaptation from an agroclimatic perspective," in Chickpea Breeding and Management, S. S. Yadav, R. J. Redden, W. Chen, and B. Sharma, Eds., pp. 47-71, CABI, Wallingford, UK, 2007.

[3] N. Katerji, J. W. van Hoorn, A. Hamdy, M. Mastrorilli, T. Oweis, and R. S. Malhotra, "Response to soil salinity of two chickpea varieties differing in drought tolerance," Agricultural Water Management, vol. 50, no. 2, pp. 83-96, 2001.

[4] H. Mhadhbi, M. Jebara, A. Zitoun, F. Limam, and M. E. Aouani, "Symbiotic effectiveness and response to mannitol-mediated osmotic stress of various chickpea-rhizobia associations," World Journal of Microbiology and Biotechnology, vol. 24, no. 7, pp. 1027-1035, 2008.

[5] T. J. Flowers, P. M. Gaur, C. L. L. Gowda et al., "Salt sensitivity in chickpea," Plant, Cell and Environment, vol. 33, no. 4, pp. 490-509, 2010.

[6] P. M. Gaur, A. K. Jukanti, and R. K. Varshney, "Impact of genomic technologies on chickpea breeding strategies," Agronomy, vol. 2, no. 3, pp. 199-221, 2012.

[7] P. M. Gaur, S. Tripathi, C. L. L. Gowda et al., Chickpea Seed Production Manual, ICRISAT, Andhra Pradesh, India, 2010.

[8] M. Kassie, B. Shiferaw, A. Solomon et al., Current Situation and Future Outlooks of the Chickpea Sub Sector in Ethiopia, International Crops Research Institute for the Semi-arid Tropics (ICRISAT), Nairobi, and Ethiopian Institute of Agricultural Research (EIAR), DeberZeitAgricultral Research Centre, DebreZeit, Ethiopia, 2009.

[9] FAO, "Food and Agriculture Organizations of the United Nations: statistics division," 2015, http://faostat.fao.org/site/ 567/default.aspx.

[10] G. Bejiga, M. Eshetu, and Y. Anbese, Improved Cultivars and Production Technology of Chickpea in EthiopiaDebre-zeit,
Ethiopia: Debre- Zeit Agricultural Research Center, Alemaya University of Agriculture, Dire Dawa, Ethiopia, 1996.

[11] G. Bejiga and K. Daba, "Breeding chickpea for wide adaptation," in Food and Forage Legumes of Ethiopia Progress and Prospects, K. Ali, G. keneni, S. Ahmed et al., Eds., pp. 59-66, ICARDA, Addis Ababa, Ethiopia, 2006.

[12] G. Keneni, E. Bekele, E. Getu, M. Imtiaz, K. Dagne, and F. Asefa, "Characterization of Ethiopian chickpea (Cicer arietinum L.) germplasm accessions for response to infestation by adzuki bean beetle (Callosobruchus chinensis L.) I. performance evaluation," Ethiopian Journal of Agricultural Science, vol. 21, pp. 65-83, 2011.

[13] K. E. Giller, Nitrogen Fixation in Tropical Cropping Systems, CABI publishing, Wallingford, UK, Second edition, 2001.

[14] H. Korir, N. W. Mungai, M. Thuita, Y. Hamba, and C. Masso, "Co-inoculation effect of rhizobia and plant growth promoting rhizobacteria on common bean growth in a low phosphorus soil," Frontiers in Plant Science, vol. 8, p. 141, 2017.

[15] M. Erman, S. Demir, E. Ocak, Ş. Tüfenkçi, F. Oğuz, and A. Akköprü, "Effects of Rhizobium, arbuscular mycorrhiza and whey applications on some properties in chickpea (Cicer arietinum L.) under irrigated and rainfed conditions 1-yield, yield components, nodulation and AMF colonization," Field Crops Research, vol. 122, no. 1, pp. 14-24, 2011.

[16] P. H. Graham, "Some problems of nodulation and symbiotic nitrogen fixation in Phaseolus vulgaris L.: a review," Field Crops Research, vol. 4, pp. 93-112, 1981.

[17] G. Hardarson, "Methods for enhancing symbiotic nitrogen fixation," in Enhancement of Biological Nitrogen Fixation of Common Bean in Latin AmericaSpringer, Dordrecht, Netherlands, 1993.

[18] P. Somasegaran and H. J. Hoben, "Handbook for rhizobia: methods in legume-Rhizobium technology," Plant and Soil, vol. 174, pp. 3-28, 2012.

[19] C. Freiberg, R. Fellay, A. Bairoch, W. J. Broughton, A. Rosenthal, and X. Perret, "Molecular basis of symbiosis between Rhizobium and legumes," Nature, vol. 387, no. 6631, pp. 394-401, 1997.

[20] M. Hungria and M. A. Vargas, "Environmental factors affecting $\mathrm{N}_{2}$ fixation in grain legumes in the tropics, with an emphasis on Brazil," Field Crops Research, vol. 65, no. 2-3, pp. 151-164, 2000.

[21] E. A. Davidson, "The contribution of manure and fertilizer nitrogen to atmospheric nitrous oxide since 1860," Nature Geoscience, vol. 2, no. 9, pp. 659-662, 2009.

[22] D. N. Suprapta, "Potential of microbial antagonists as biocontrol agents against plant fungal pathogens," Journal of ISSAAS, vol. 18, no. 2, pp. 1-8, 2012.

[23] EARO (Ethiopian Agricultural Research Organization, Crop Research Strategy Stat.Bulletin), EARO (Ethiopian Agricultural Research Organization, Addis Abeba, Ethiopia, 2000.

[24] G. Agegnehu and R. Fessehaie, "Response of faba bean to phosphate fertilizer and weed control on nitisols of Ethiopian highlands," Italian Journal of Agronomy, vol. 1, no. 2, pp. 281-290, 2006.

[25] H. H. Zahran, "Conditions for successful Rhizobium-legume symbiosis in saline environments," Biology and Fertility of Soils, vol. 12, no. 1, pp. 73-80, 1991.

[26] Z. Belay and F. Assefa, "Symbiotic and phenotypic diversity of Rhizobium leguminosarumbv. viciae from Northern Gondar, Ethiopia," African Journal of Biotechnology, vol. 10, pp. 4372-4379, 2011. 
[27] M. Jida and F. Assefa, "Phenotypic diversity and plant growth promoting characteristics of Mesorhizobium species isolated from chickpea (Cicer arietinum L.) growing areas of Ethiopia," African Journal of Biotechnology, vol. 11, no. 29, pp. 7483-7493, 2012.

[28] F. Shemikite, "Pattern of nodulation and nitrogen fixing performance of introduced forage legumes of some parts of North Gondar, Ethiopia," M.sc. Thesis, Addis Ababa University, Addis Ababa, Ethiopia, 2009.

[29] Z. Belay, "Symbiotic and phenotypic diversity of Rhizobium Leguminosarumvar isolates (Viciafaba) from Northern Gonder," M.SC. Thesis, Addis Ababa University, Addis Ababa, Ethiopia, 2006.

[30] D. Muleta, "Phenotypic and symbiotic effectiveness characterization of rhizobia nodulating chick pea (Cicer arietinum L.) from some parts of Ethiopia," M.sc. Thesis, Addis Ababa University, Addis Ababa, Ethiopia, 2009.

[31] F. Assefa, C. Ojewo, and S. G. Turoop, "Symbiotic effectiveness of elite rhizobia strains nodulating Desi type of chickpea (Cicer aeietinum L.) varieties," Journal of Plant Sciences, vol. 4, no. 4, pp. 88-94, 2016.

[32] T. Ereso, "Symbiotic effectiveness of rhizobia from chickpea (Cicer arietinum L.) and, phenotypic and symbiotic characterization of rhizobia nodulating faba bean (Vicia faba L.) from Southern Ethiopia," Research Journal of Microbiology, vol. 7, pp. 280-296, 2017.

[33] M. Adal, F. Assefa, and M. Eshetu, "Diversity, symbiotic and plant growth promoting properties of rhizobia and rhizobacteria of grass pea (Lathyrus sativus L.) from Central Ethiopia: implication to the selection and use of microbial inoculants in low input agriculture in Ethiopia," Doctoral Dissertation, Addis Ababa University, Addis Ababa, Ethiopia, 2018.

[34] S. Ahmed and F. Assefa, "Symbiotic effectiveness and phenotypic characterization of rhizobia nodulating chickpea (Cicer arietinum) from some growing areas of South and North wello zones," MSc. Thesis, Department of Microbial Cellular and Molecular Biology, Addis Ababa University, Addis Ababa, Ethiopia, 2010.

[35] P. Somasegaren and H. J. Hoben, "Hand book for rhizobia," Methods in Legume-Rhizobium Technology, pp. 1-441, Springer verlag, New York, NY, USA, 1994.

[36] J. M. Vincent, A Manual for the Practical Study of the RootNodule Bacteria, Blackwell Scientific Publications, Oxford, UK, 1970.

[37] J. G. Holt, N. R. Krieg, P. H. A. Sneath, J. T. Staley, and S. T. Williams, "Group 5, facultatively anaerobic gram-negative rods," in Bergey's manual of determinative bacteriology, pp. 175-289, William and Wilkins, Baltimore, MD, USA, 9th edition, 1994.

[38] J. D. Buck, "Nonstaining (KOH) method for determination of gram reactions of marine bacteria," Applied and Environmental Microbiology, vol. 44, no. 4, pp. 992-993, 1982.

[39] N. Z. Lupwayi and I. Haque, "Legume-Rhizobium technology manual," ILCA Environmental Sciences Working Document (ILCA), ILCA, Tokyo, Japan, 1994.

[40] E. Martínez-Romero, L. Segovia, F. M. Mercante, A. A. Franco, P. Graham, and M. A. Pardo, "Rhizobium tropici, a novel species nodulating Phaseolus vulgaris L. beans and Leucaena sp. trees," International Journal of Systematic Bacteriology, vol. 41, no. 3, pp. 417-426, 1991.

[41] D. C. Jordan, "Bradyrhizobium," Bergey's Manual of Systematic Bacteriology, vol. 1, pp. 242-244, 1984.
[42] D. White, The Physiology and Biochemistry of Prokaryotes, Oxford University Press, Oxford, UK, 1995.

[43] P. Somasegaran and H. J. Hoben, Hand Book for Rhizobia-Methods in Legume RhizobiumTechnology, SpringerVerlag, Heidelberg, Germany, 1984.

[44] Mulongoy, K. Technical paper 2: Biological nitrogen fixation. 2004; 1-3.

[45] J. L. Gao, J. G. Sun, Y. Li, E. T. Wang, and W. X. Chen, "Numerical taxonomy and DNA relatedness of tropical rhizobia isolated from hainan province, China," International Journal of Systematic Bacteriology, vol. 44, no. 1, pp. 151-158, 1994.

[46] G. Bernal and P. H. Graham, "Diversity in the rhizobia associated with Phaseolus vulgaris L. in Ecuador, and comparisons with Mexican bean rhizobia," Canadian Journal of Microbiology, vol. 47, no. 6, pp. 526-534, 2001.

[47] M. Hungaria, D. S. Andrade, L. M. Chueira, A. Probanza, F. J. Guttierrez-Mañero, and M. Megías, "Isolation and characterization of new efficient and competitive bean (Phaseolus vulgaris L.) rhizobia in Brazil," Soil Biology and Biochemistry, vol. 32, no. 11-12, pp. 1515-1528, 2000.

[48] A. W. Bauer, W. M. Kirby, J. C. Sherris, and M. Turck, "Antibiotic susceptibility testing by a standardized single disk method," American Journal of Clinical Pathology, vol. 45, no. 4, pp. 493-496, 1966.

[49] R. Mhamdi, M. Jebara, M. E. Aouani, R. Ghrir, and M. Mars, "Genotypic diversity and symbiotic effectiveness of rhizobia isolated from root nodules of Phaseolus vulgaris L. grown in Tunisian soils," Biology and Fertility of Soils, vol. 28, no. 3, pp. 313-320, 1999.

[50] M. H. Abd-Alla, F. M. Morsy, A.-W. E. El-enany, and T. Ohyama, "Isolation and characterization of a heavy-metalresistant isolate of Rhizobium leguminosarum bv. viciae potentially applicable for biosorption of $\mathrm{Cd}^{2+}$ and $\mathrm{Co}^{2+}$," International Biodeterioration \& Biodegradation, vol. 67, pp. 48-55, 2012.

[51] N. Amarger, V. Macheret, and G. Laguerre, "Rhizobium gallicum sp. nov. and Rhizobium giardinii sp. nov., from Phaseolus vulgaris nodules," International Journal of Systematic Bacteriology, vol. 47, no. 4, pp. 996-1006, 1997.

[52] S. M. Nour, M. P. Fernandez, P. Normand, and J.-C. CleyetMarel, "Rhizobium ciceri sp. nov., consisting of strains that nodulate chickpeas (Cicer arietinum L.)," International Journal of Systematic Bacteriology, vol. 44, no. 3, pp. 511-522, 1994.

[53] B. Aynalem and F. Assefa, "Effect of glyphosate and mancozeb on the rhizobia isolated from nodules of Vicia faba L. and on their N2-fixation, North Showa, Amhara Regional State, Ethiopia," Advances in Biology, vol. 2017, Article ID 5864598, 2017.

[54] A. K. Singh and B. Meenakshi, "Characterization of Mesorhizobium sp. isolated from root nodules of Cicer arietinum," International Journal of Agricultural Science and Research (IJASR), vol. 2, no. 3, pp. 142-154, 2012.

[55] M. A. Mohammed, M. T. Chernet, and F. A. Tuji, "Phenotypic, stress tolerance, and plant growth promoting characteristics of rhizobial isolates of grass pea," International Microbiology, vol. 23, no. 4, pp. 607-618, 2020.

[56] K. B. Stephen, E. Alfred, A. L. Slinkard, and L. W. Fran, "LEGUMES: evaluation of rhizobial inoculation methods for chickpea," Agronomy Journal, vol. 94, no. 4, pp. 851-859, 2002.

[57] J. Brockwell, R. Gault, D. Herridge, L. Morthorpe, and R. Roughley, "Studies on alternative means of legume 
inoculation: microbiological and agronomic appraisals of commercial procedures for inoculating soybeans with Bradyrhizobium japonicum," Australian Journal of Agricultural Research, vol. 39, no. 6, pp. 965-972, 1988.

[58] M. Sahgal and B. N. Johri, "The changing face of rhizobial systematics," Current Science, vol. 84, no. 1, pp. 43-48, 2003.

[59] M. B. Peoples, D. F. Herridge, and J. K. Ladha, "Biological nitrogen fixation: an efficient source of nitrogen for sustainable agricultural production?" Plant and Soil, vol. 174, no. $1 / 2,1995$.

[60] M. Jida and F. Assefa, "Phenotypic and plant growth promoting characteristics of Rhizobium leguminosarumbv. viciae from lentil growing areas of Ethiopia," African Journal of Microbiology Research, vol. 5, no. 24, pp. 4133-4142, 2011.

[61] C. Kucuk, "In vitro antagonism of Rhizobium strains isolated from various legumes," JABS, vol. 7, pp. 24-30, 2013.

[62] D. R. Graham and D. Jeffrey, "Citrobacter diversus brain abscess and meningitis in neonates," Journal of the American Medical Association, vol. 245, no. 19, pp. 1923-1925, 1981.

[63] P. H. Graham, "Stress tolerance in Rhizobium and Bradyrhizobium, and nodulation under adverse soil conditions," Canadian Journal of Microbiology, vol. 38, no. 6, pp. 475-484, 1992.

[64] A. Sessitsch, H. Ramirez-saad, G. Hardarson, A. D. L. Akkermans, and W. M. De Vos, "Classification of Austrian rhizobia and the Mexican isolate FL27 obtained from Phaseolus vulgaris L. as Rhizobium gallicum," International Journal of Systematic Bacteriology, vol. 47, no. 4, pp. 10971101, 1997.

[65] G. Kenasa, M. Jida, and F. Assefa, "Characterization of phosphate solubilizing faba bean (Vicia faba L.) nodulating rhizobia isolated from acidic soils of Wollega, Ethiopia," Science, Technology and Arts Research Journal, vol. 3, no. 3, pp. 11-17, 2014.

[66] G. Tesfaye and F. Assefa, "Symbiotic and phenotypic diversity of rhizobial isolates nodulating Faba bean (Vicia faba L.) from Western Shoa and Western Hararghe, Ethiopia," MSc Thesis, School of Graduate Studies, Addis Ababa University, Addis Ababa, Ethiopia, 2008.

[67] A. Beshah and F. Assefa, "Isolation, identification and characterization effective rhizobium species nodulating mung bean (Vigna radiata) from some places of North shewa," International Journal of Environmental Sciences \& Natural Resources, vol. 20, no. 1, Article ID 556026, 2019.

[68] Ç. Küçük and M. Kıvanç, "Preliminary characterization of Rhizobium strains isolated from chickpea nodules," African Journal of Biotechnology, vol. 7, no. 6, pp. 772-775, 2008. 